INPLASY

PROTOCOL

To cite: Sui et al. Meta-analysis of curative effect of Sakubatrovalsartan combined with Qiliqiangxin capsule in the treatment of patients with chronic cardiac failure. Inplasy protocol 202130115. doi: 10.37766/inplasy2021.3.0115

Received: 31 March 2021

Published: 31 March 2021

Corresponding author: YanBo Sui

shawn.wye@gmail.com

Author Affiliation: HeiLongJiang University Of Chinese Medicine.

Support: NNSFC: 81904107.

Review Stage at time of this submission: Data analysis.

Conflicts of interest:

None declared.

\section{Meta-analysis of curative effect of Sakubatrovalsartan combined with Qiliqiangxin capsule in the treatment of patients with chronic cardiac failure}

Sui, YB1; Wei, JX2; Song, C33 Zheng, XH4; Yang, SZ5.

Review question / Objective: The purpose of this study is to compare the efficacy, cardiac function, serological index, exercise endurance, safety and other indexes of RCT test in patients with chronic heart failure by comparing the efficacy, cardiac function, serological indexes, exercise endurance, safety and other indexes in the RCT test of ShakubaTrue valsartan combined with Qiliqiangxin capsule and shakubar valsartan alone, in order to further evaluate the efficacy and safety of Shakubatrextan combined with Qiliqiangxin capsule. Condition being studied: In recent years, many studies have shown that in the conventional treatment of CHF, sacubitril and valsartan combined with Qiliqiangxin capsules have better intervention effects than simple sacubitril and valsartan. However, there are also a few studies that suggest that this type of treatment is better. Compared with integrated western medicine treatment, its advantages are not outstanding.

INPLASY registration number: This protocol was registered with the International Platform of Registered Systematic Review and Meta-Analysis Protocols (INPLASY) on 31 March 2021 and was last updated on 31 March 2021 (registration number INPLASY202130115).

\section{INTRODUCTION}

Review question / Objective: The purpose of this study is to compare the efficacy, cardiac function, serological index, exercise endurance, safety and other indexes of RCT test in patients with chronic heart failure by comparing the efficacy, cardiac function, serological indexes, exercise endurance, safety and other indexes in the RCT test of ShakubaTrue valsartan combined with Qiliqiangxin capsule and shakubar valsartan alone, in order to further evaluate the efficacy and 
safety of Shakubatrextan combined with Qiliqiangxin capsule.

Condition being studied: In recent years, many studies have shown that in the conventional treatment of $\mathrm{CHF}$, sacubitril and valsartan combined with Qiliqiangxin capsules have better intervention effects than simple sacubitril and valsartan. However, there are also a few studies that suggest that this type of treatment is better. Compared with integrated western medicine treatment, its advantages are not outstanding.

\section{METHODS}

Search strategy: Chinese search terms include：心力衰竭、心衰、慢性心力衰竭、慢 性心衰、心功能不全、胸痹、胸痛、心痛、短 气、CHF、沙库巴曲缴沙坦、ARNI、芪劳强心 胶囊、随机; . English search terms include: (Take Pubmed as an example) \#1 "Heart Failure"[Mesh] \#2 "Heart Failure" OR "chronic heart failure" OR "Cardiac Failure" OR "diastolic heart failure" OR "heart failure with normal ejection fraction" OR "heart failure with preserved ejection fraction" OR "heart failure with reduced ejection fraction" OR "ventricular dysfunction" OR "LV dysfunction" OR "left ventricular systolic dysfunction" OR "left ventricular diatolic dysfunction" OR "Heart Decompensation" OR "Decompensation, Heart" OR "Heart Failure, Right-Sided" OR "Heart Failure, Right Sided" OR "RightSided Heart Failure" OR "Right Sided Heart Failure" OR "Myocardial Failure" OR "Congestive Heart Failure" OR "Heart Failure, Congestive" OR "Heart Failure, Left-Sided" OR "Heart Failure, Left Sided" OR "Left-Sided Heart Failure" OR "Left Sided Heart Failure" OR "HFNEF" OR "HFPEF" OR "HFREF" OR "HF NEF" OR "HF PEF" OR "HF REF"[Title/Abstract]; \#3 \# 1 OR \# 2 ; \# 4 "s acubitril valsartan" [Supplementary Concept]; \#5 "qiliqiangxin" [Supplementary Concept]; \#6 \#3 AND \#4 AND \#5.

Participant or population: Patients with heart failure diagnosed by the guidelines for the diagnosis and treatment of Heart failure in China. There are no limits to research subjects' age, gender, race, condition duration or intensity.

Intervention: Under routine treatment, the experimental group was treated with Sakubatrovalsartan combined with Qiliqiangxin capsule in the treatment of heart failure.

Comparator: In the control group, patients with heart failure were treated with Sakubatrovalsartan under routine treatment.

Study designs to be included: Only randomized controlled clinical trials (RCTs) related to the efficacy of Sakubatrovalsartan combined with Qiliqiangxin capsule in the treatment of chronic heart failure will be included in this systematic review. Trials published in the form of dissertations will be also selected as eligible studies.

Eligibility criteria: Interventions included the use of Sakubatrovalsartan and Qiliqiangxin capsules under any type of conventional treatment for patients with chronic heart failure.

Information sources: Relevant studies will be searched from the databases of PubMed, Embase, Cochrane Library, China Knowledge Resource Integrated Database, Weipu Database for Chinese Technical Periodicals, SinoMed, and WanFang Database.

Main outcome(s): Effectiveness, N-terminal pro-brain natriuretic peptide (NT-proBNP), Left ventricular ejection fraction (LVEF), Left ventricular end-diastolic diameter (LVEDD), 6-minute walking distance (6MWD), Cardiac output (CO), Left Ventricular end-systolic diameter (LVESD), and Safety.

Quality assessment / Risk of bias analysis: We will use the Cochrane Collaboration tool which is recommended by the Cochrane Reviewer's Handbook to assess the risk of bias for quality assessment of 
the included studies. The studies will be graded based on I. random sequence generation; II. allocation concealment; III.blinding; IV.incomplete outcome data; V. selective outcome reporting; VI. other sources of bias.

Strategy of data synthesis: To combined the data of the experimental group was treated with Sacubarovsartan combined with Qiliqiangxin Capsules, while the control group had no use of the Qiliqiangxin Capsules. The data of the study included may be divided into two cases, depending on whether the data are suitable for meta-analysis. If the metaanalysis will not be performed because of heterogeneity, interventions, comparisons outcomes, etc. we will make forms for a qualitative description. If the data is suitable for meta-analysis, we will perform the meta-analysis using software Stata 16.1. For dichotomous data, we will present the results as risk ratios with $95 \%$ confidence intervals (Cls). For continuous data, the mean difference (MD) will be presented. If outcome variables are measured on different scales, standard mean differences (SMD) analysis with 95\% Cls will be performed. For the data will be done with the meta-analysis, the heterogeneity will be tested by a standard $I^{2}$ test. If there is no statistical heterogeneity among the results, the fixed effects model is employed for metaanalysis. If there is a statistic heterogeneity, the source of the heterogeneity should be further analyzed. If there is obvious clinical heterogeneity, the subgroup or sensitivity analysis, or only descriptive analysis can be performed.

Subgroup analysis: The subgroup study was carried out according to the intervention time < 4 weeks, 4-12 weeks, and $>12$ weeks.

Sensitivity analysis: The sensitivity analysis is carried out by using Stata software, and the sensitive situation of the article is reflected by deleting the change of the amount of effect after a certain article is deleted.
Language: Without any language or publication status restrictions.

\section{Country(ies) involved: China.}

Other relevant information: When data are missing, we will look for the reason. Then we will contact the corresponding author to obtain and verify the data if possible. If this does not work, we will only analyze the available data.

Keywords: Heart failure, ARNI, Sakubatrovalsartan, Qiliqiangxin capsule, Systematic review, Meta-analysis, Randomized controlled trial.

Contributions of each author:

Author 1 - YanBo Sui.

Author 2 - JinXuan Wei.

Author 3 - Chao Song.

Author 4 - XiaoHang Zheng.

Author 5 - SuZhen Yang. 